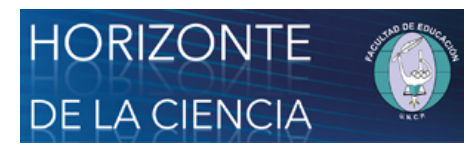

Horizonte de la Ciencia

ISSN: 2413-936X

horizontedelaciencia@uncp.edu.pe

Universidad Nacional del Centro del Perú

Perú

\title{
Desafíos del modelo colombiano pedagógico para las habilidades lectoras en primer grado de la educación básica primaria
}

Chalá Molina, Betty; Pérez Padrón, María Caridad

Desafíos del modelo colombiano pedagógico para las habilidades lectoras en primer grado de la educación básica primaria

Horizonte de la Ciencia, vol. 10, núm. 18, 2020

Universidad Nacional del Centro del Perú, Perú

DOI: https://doi.org/10.26490/uncp.horizonteciencia.2020.18.403

Los autores otorgan el permiso a compartir y usar su trabajo manteniendo la autoría del mismo. Atribución no comercial (CC BY-NC) 4.0 


\section{Desafíos del modelo colombiano pedagógico para las habilidades lectoras en primer grado de la educación básica primaria}

Challenges of the Colombian pedagogical model for reading skills in first grade of primary basic education

Desafios do modelo pedagógico colombiano para as habilidades de leitura na primeira série do ensino fundamental

Colombiap yachachini likanapnu atipayninkuna,ñawinchay yachaykunapa yachaywasichu yachapakuy allakukuna

ANTSAINKIMATERO OSHIYAKABENTARI COLOMBIANO IBAMETANTERI

ASHIMITSATSIMOTERINE NAANATATSINE APARONI OSARENTSI YOMETANTSIKI

Kengagantsi ikogi irosikotanërini Colombia kara ojigotagani inibabasiti obatatsi isangenaigi janekijanipage kara jigotagantsikë

Betty Chalá Molina Datos de las autoras

Institución Educativa Nuevo Latir, Colombia

DOI: https://doi.org/10.26490/

bettychala515@gmail.com

uncp.horizonteciencia.2020.18.403

(iD http://orcid.org/0000-0002-8546-6138

María Caridad Pérez Padrón

Universidad Carlos Rafael Rodríguez de Cienfuegos, Cuba

mcperez@ucf.edu.cu

iD http://orcid.org/0000-002-0938-0578

Recepción: 25 Febrero 2019

Corregido: 11 Julio 2019

Aprobación: 20 Agosto 2019

Recepción: 25 Febrero 2019

Corregido: 11 Julio 2019

Aprobación: 20 Agosto 2019

\section{RESUMEN:}

El objetivo del presente artículo es indagar sobre los desafíos para el desarrollo de las habilidades lectoras en el primer ciclo en la Educación Básica Primaria. Se parte de la caracterización de las habilidades lectoras en la Educación Básica Primaria en Colombia. La metodología aplicada consistió en la revisión de documentos normativos, como el Proyecto Educativo Institucional y planes de aula; entrevistas y encuestas a docentes y directivos y una prueba pedagógica a escolares de primer grado. Se indagó sobre la correspondencia entre las indicaciones de los documentos normativos y su concreción en los proyectos educativos institucionales en Cali, su puesta en práctica y resultados en los escolares. Los resultados alcanzados revelan una contradicción en cuanto al modelo educativo colombiano sobre el desarrollo de las habilidades lectoras a nivel nacional y la realidad de la práctica educativa; además, se evidenciaron las diferencias significativas cuando se introduce la actividad lúdica.

Palabras ClaVe: Habilidades lectoras, comprensión, métodos, diagnóstico, lúdica, Nawinchay yachaykuna, mayay, lulaykuna, lisipana, awsaynilakuna, ashimitsatsimoterine ñanatatsine, kematsatakotzirori, aretakotyari, kobityaperotatsiri, ñatsatzinkari, ogotagantsi, nibabasitagantsi, ogotagero, anetsanakero, magempitagantsi.

\section{Abstract:}

\section{NotAS DE AUTOR}

Datos Betty Chalá Molina. Investigadora y docente de aula en Básica primaria, Licenciada en Básica primaria con énfasis en Ciencias Sociales. Aspirante de las a Doctor en Ciencias Pedagógicas de la Universidad Carlos Rafael Rodríguez de Cienfuegos Ciudad de Cienfuegos. Especialista en Gerencia Social autoras de la Pontificia Universidad Javeriana. De Cali- Colombia.

María Caridad Pérez Padrón. Licenciada en español y Literatura de la Universidad Central Martha Abreu de las Villas. Doctora en Ciencias Pedagógicas de la Universidad Carlos Rafael Rodríguez de Cienfuegos

bettychala515@gmail.com 
The objective of this article is to investigate the challenges for the development of reading skills in the first cycle in Primary Basic Education. It is based on the characterization of reading skills in Primary Basic Education in Colombia. The methodology applied consisted in the revision of institutional documents, such as the Institutional Educational Project and classroom plans; interviews and surveys to teachers and managers and a pedagogical test to first grade students. The correspondence between the indications of the normative documents and their concretion in the institutional educational projects in Cali was investigated, as well as their implementation and results achieved in the students. The results achieved reveal a contradiction regarding the Colombian educational model on the development of reading skills at the national level and the reality of educational practice; In addition, significant differences were evidenced when the playful activity is introduced.

KEYWORDS: Reading skills, comprehension, methods, diagnosis, playful.

\section{Resumo:}

O objetivo deste artigo é indagar os desafios para o desenvolvimento das habilidades leitoras no primeiro ciclo do ensino fundamental. Baseia-se na caracterização das habilidades de leitura no ensino básico da Colômbia. A metodologia aplicada consistiu na revisão de documentos normativos, como o Projeto Educacional Institucional e os planos de aula; entrevistas e enquetes com professores e equipe diretiva e uma prova pedagógica para as crianças do primeiro ano escolar. Indagou-se sobre a correspondência entre as indicações dos documentos normativos e sua concretização nos projetos educacionais institucionais em Cali, sua implementação e resultados nos estudantes. Os resultados alcançados revelam uma contradição em relação ao modelo educacional colombiano sobre o desenvolvimento das habilidades de leitura em nível nacional e a realidade da prática educacional; além disso, se evidenciaram as diferenças significativas quando se introduz a atividade lúdica.

Palavras-chave: habilidades leitoras, compreensão, métodos, diagnóstico, lúdico.

\section{INTRODUCCIÓN}

Las condiciones y la estratificación socioeconómica del capitalismo global, el creciente desarrollo de la tecnología y las comunicaciones, han llevado a que los niños vivan aceleradamente procesos de aprendizajes externos a la escuela y a la familia. El Proceso de Enseñanza-Aprendizaje de la escuela primaria, exige interpretar realidades, derivadas de un mundo interconectado, dinámico, vivo, biodiverso, natural y necesitado de ser comprendido, en particular por los niños. Es una tarea apremiante que a la niñez se le ofrezca la oportunidad de ser crítica y adquirir habilidades; que le permitan leer las manifestaciones de un mundo globalizado, y lo prepare para la participación activa en la sociedad.

La asignatura Lengua Castellana tiene una función determinante en la Educación Básica Primaria, porque a partir de sus contenidos contribuye al desarrollo de las habilidades comunicativas y propicia la ampliación del campo de experiencias del escolar. Para lograr lo anterior, es necesario que estos desarrollen las macrohabilidades de hablar, escuchar, leer y escribir, (Cassany, Luna y Sanz, 1998); de allí, que diversos especialistas han centrado su atención en el proceso de enseñanza aprendizaje de la lectura.

Al respecto, Balmaseda, (2012, p.52) plantea como uno de los desafíos la necesidad de desarrollar habilidades en el uso de la lengua y afirma que la lectura y la escritura en las sociedades letradas, son condiciones esenciales integrarse a la sociedad con el dominio de estos cognitivos complejos. De allí, la importancia de vincular al proceso de enseñanza aprendizaje de la lectura y la escritura, estrategias que comprometan la lúdica para llevar al escolar a adquirir el hábito de la lectura y su goce, al punto de asumir que ésta forma parte de su vida cotidiana.

Las experiencias de aula muestran la utilidad de los métodos lúdicos, para la formación de habilidades lectoras. (Farías y Rojas, 2010) ; (Delgadillo y Chacón, 2014); Castro, Robledo y Tello (2017). Otras experiencias en prácticas de aula para la enseñanza de la lectura comprensiva mediante el análisis literal e inferencial del texto, involucran algunos elementos audiovisuales que aportan al Proceso de EnseñanzaAprendizaje, lo que diversifica los medios de enseñanza, aun así, se evidencia la falta de seguimiento de esta, en los procesos de lectura, la poca participación de los escolares en la clase y las dificultades encontradas desde la organización curricular (García, 2002;Barboza, Delhi, y Peña, 2014). 
Por tal razón, el objetivo del presente artículo es indagar sobre los desafíos que implican en el desarrollo de las habilidades lectoras en el primer ciclo en la Educación Básica Primaria.

\section{El programa Lengua Castellana del primer CiClo de EDUCACión básica PRimaria en Colombia}

En Colombia la Educación Básica Primaria, inicia en el primer ciclo, que comprende de primero a tercer grado; este ciclo se enfoca hacia los conocimientos básicos en las asignaturas como lectura, escritura, aritmética y competencias ciudadanas. La edad de ingreso de los escolares es entre los cinco y los siete años y la duración de la escolarización es de tres años.

En concordancia con la Ley General de Educación Colombiana de 1994, el desarrollo de la organización por ciclos se norma mediante la Resolución 2343 de 1996 "Por la cual diseñan los lineamientos generales de los procesos curriculares del servicio público educativo y se establecen los indicadores de logros curriculares para la educación formal” (p.26).

El Ministerio de Educación Nacional de Colombia en pro de la calidad de la educación se traza como uno de los propósitos, la flexibilización pertinente de los contenidos, con lo que el escolar desea y debe aprender. Asimismo, se busca centralizar el desarrollo evolutivo del escolar como referente para la modificación de las formas de evaluación, tanto en sus propósitos, como en sus metodologías. (MEN, 2006).

Los lineamientos curriculares de la lengua castellana (MEN, 2006), en cumplimiento del artículo 78 de 1991 de la constitución colombiana; parten de los criterios: qué enseñar y para qué enseñar. Estos se crean en procesos históricos que fueron determinantes en Colombia, como los movimientos pedagógicos y de concordancia con la Ley General de Educación y la instauración de los proyectos educativos institucionales.

En los lineamientos curriculares (MEN, 2006) se manifiesta la esencia de la Lengua Castellana como la agrupación de signos y reglas de uso patrimonial; más no se refieren a la actividad lúdica como manifestación social de dicho patrimonio ni tampoco es tenida en cuenta para la formación de las habilidades lectoras. Para tal fin, el documento declara siete competencias: literaria, textual, gramatical, enciclopédica, poética, pragmática y semántica, con las cuales se orienta el Proceso de Enseñanza-Aprendizaje de la lengua castellana.

$\mathrm{Al}$ analizar los lineamientos curriculares, se observa la necesidad de la formación de las habilidades lectoras en los escolares debido a que antes de alcanzar una competencia se debe formar las habilidades en estos. En particular, la macrohabilidad de leer, la cual es declarada también en los lineamientos curriculares.

De igual manera, se puntualiza la importancia de los lineamientos curriculares como los derroteros generales orientadores en el Proceso de Enseñanza-Aprendizaje de la Lengua Castellana, sin embargo, para aplicar en la práctica el modelo contenido en los lineamientos se requiere hacer una contextualización, que se adecue a las condiciones concretas de los escolares, los docentes y las instituciones educativas en que se aplique dicho modelo.

El Ministerio de Educación Nacional de Colombia para facilitar la labor del maestro publicó los Estándares Básicos de Competencias de Lengua Castellana. Estos son definidos en el año 2006 como el conjunto de capacidades para el desarrollo del lenguaje para la formación del individuo y la consolidación de la sociedad. La esencia de este modelo es lograr la competencia lingüística que posibilite el desempeño en la vida, como individuos miembros de un grupo social. Estos documentos presentan coherencia de manera vertical, para el mismo grado y grupo por conjuntos de grado, así como también en la horizontalidad de los ejes; corresponden a la producción textual, comprensión e interpretación textual, literatura, medios de comunicación y otros sistemas simbólicos y ética de la comunicación.

De modo, que los documentos anteriormente mencionados, contienen el modelo por competencias que debe considerarse para diseñar la estrategia didáctica de lectura en cada institución educativa. Se propone como aspecto fundamental hacer uso de los ejes que corresponden a la lectura. 
Se cuenta, además, con los Derechos Básicos de Aprendizaje DBA (MEN, 2016). Estos contienen los tipos de conocimientos que el escolar debe manejar, a lo largo de un grado específico. Este documento es un instrumento de trabajo del docente para el diseño del proceso de enseñanza aprendizaje orientado a que los escolares dominen las habilidades relacionadas con la lectura. En los contenidos, se encuentran las cuatro macrohabilidades comunicativas: leer, escribir, escuchar y hablar. Sin embargo, en los enunciados de los DBA, se muestran pocas situaciones comunicativas relacionadas con la actividad lúdica que orienten al docente como en el diseño para concretar el proceso de enseñanza aprendizaje, en particular, en las tareas docentes para despertar en los estudiantes la curiosidad e interés personal con el fin de lograr los conocimientos en correspondencia con los resultados esperados.

La Malla de aprendizaje de Lenguaje (2015) es un manual didáctico que articula las competencias lingüísticas en el escolar de grado primero. Esta contiene los saberes y habilidades que debe alcanzar el escolar durante su año escolar. Las mallas se encuentran interrelacionadas con los DBA. En las mallas de aprendizaje se pueden ver, cuáles son los mínimos cognitivos que el escolar debe adquirir en consonancia con las cuatro macrohabilidades: leer, escuchar, hablar, escribir.

Por esta razón, desde la Malla de aprendizaje de Lenguaje, se orienta a los docentes para que propicien el acceso a la cultura escrita reconociendo la importancia de leer como la habilidad que se fundamenta a partir de la decodificación por medio de la conciencia fonológica, el conocimiento del alfabeto, la ampliación del vocabulario y el reconocimiento de textos. Es necesario potencializar, que aunque es un documento reciente, se sostiene en presupuestos tradicionales.

El Proyecto Educativo Institucional como modelo pedagógico de la institución educativa debe concretar las recomendaciones y exigencias del currículo dada en los documentos de carácter nacional. Como parte de este proyecto están también los planes de aulas en los cuales el maestro planifica el proceso concreto de enseñanza aprendizaje que ejecutará en su clase.

Los docentes cuentan además con el libro de texto de lectura y composición en español (2015), que es el medio de trabajo, fundamental utilizarlo en el aula para el aprendizaje de la lectura y la escritura. En este se encuentra una secuencia del contenido y ofrece instrucciones mínimas para el maestro, aunque los fines del texto están acorde a los estándares y competencias, son muy generales, justo en el momento en que el escolar inicia el reconocimiento de los signos gráficos. En el caso de grado primero, se equipara la enseñanza de la lectura con ejercicios de escritura, la motricidad fina, que no representa el acto de pensar y producir textos y queda un porcentaje mínimo a la enseñanza de la lectura. Por ejemplo, de 10 actividades de una unidad del libro de texto, solo cuatro se relacionan con la enseñanza de la comprensión de textos escritos, lo que representa la producción de las ideas y la resolución de situaciones cotidianas.

En el análisis de textos se presentan rondas tradicionales, adivinanzas y trabalenguas de manera escasa, lo que se requiere revisar y poner en consideración con los reales propósitos de la clase y proponer más variedad de literatura infantil. Este requerimiento queda a la iniciativa del docente.

En la malla curricular institucional (2015), mediante un formato de análisis de documentos se plantea la organización de clase por medio de guías de aprendizaje que indican el modo de lo que se va a enseñar, a partir del objetivo general y los objetivos específicos y las temáticas a desarrollar. En este análisis se pudo valorar que los objetivos no siempre están enfocados desde la perspectiva del escolar, sino en función de la actividad del profesor, son los casos concretos de fomentar, fortalecer, adquirir y dar un uso. Tampoco están redactados de modo tal, que contengan la habilidad a formar. Este análisis se realiza sobre la base de lo declarado por los especialistas de la didáctica que aseveran que el objetivo guía, conduce el proceso de enseñanza-aprendizaje, por lo que es necesaria su redacción clara, que delimite adecuadamente la intención.

En este documento, no aparece de manera explícita el trabajo con las habilidades lectoras para encauzar su formación desde la enseñanza de la Lengua Castellana. No se declara el método de la enseñanza de la lectura, es decir en los planes de trabajo no se ve con claridad la validación de los componentes de la lectura: fonético, semántico, visual y las estrategias a utilizar antes, durante y después de la lectura. 
En este mismo orden de ideas, al analizar los planes de aula del ciclo uno, con un total de once planes de aula, se observa que los profesores realizaron la planeación de manera general, se evidencia que no se tienen en cuenta las exigencias de los documentos oficiales, que no se definen las estrategias empleadas y que no se presenta con claridad la relación objetivo-método y estrategias.

La mayor dificultad se encuentra en el seguimiento de los planes de aula a través de la observación a clase y el desarrollo de los aprendizajes logrados por los escolares; se identifica que las tareas son más centradas hacia la escritura o la transcripción, pocas veces se definen con ejercicios de lectura, de juegos de palabras, o de reconocimiento vocálico y fonético, reconocimientos de las palabras para crear textos orales y su comprensión. Por esta razón, se valora someramente el resultado obtenido por el escolar, sin poder verificar claramente el nivel de lectura en que se encuentra, es de decir las habilidades lectoras adquiridas ni sus intereses lectores.

Al valorar los reportes de investigaciones relacionadas con la evaluación de los aprendizajes en Colombia, hay dos decretos que reglamentan este aspecto. El Decreto 230 de 2002, donde se apela a la valoración del aprendizaje por procesos y se promueve el aprendizaje por ciclos del desarrollo evolutivo, se apoya a partir de la promoción automática, que no ha sido aplicada debidamente porque se considera que menos del $5 \%$ puede ser reprobado, no importa los resultados de aprendizaje. Y el Decreto 1290 de 2009, resultado de la derogación del anterior. Este reglamenta los criterios de evaluación de manera autónoma e institucional y la promoción de los estudiantes se da a partir de cada grado cursado, poniéndose énfasis en los resultados de los aprendizajes y en los contenidos adquiridos. Los profesores que han participado de este modelo, han sido parte de la evaluación, que muestra resultados en las pruebas de Estado, por ejemplo, entre el año 2009 y 2012 , los grados $5^{\circ}$ y $9^{\circ}$ mejoraron en relación con cada una de las áreas evaluadas y la de mayor puntaje fue Lenguaje.

Además, se solicita capacitación sobre conocimientos del proceso evolutivo de los escolares y los procesos de la lectura, ya que en las instituciones educativas se dan importantes casos de maestros que asumen el primer grado por carga académica. Generalmente, no tienen experiencia en la enseñanza de la lectura en dicho grado, pues provienen de otros grados escolares de la básica primaria. (Cajiao et al., 2006).

En este primer ciclo, las prácticas a transformar se localizan en la concepción del tratamiento metodológico en la enseñanza de lengua castellana con actividades de aprestamiento ojo-mano a partir de ejercicios mecánicos hasta lograr el aprendizaje esperado. Conocimientos y habilidades que debe manejar el docente encargado de enseñarlo porque los escolares deben ser sujetos de derecho y la escuela debe ofrecer una enseñanza pertinente a todos los estratos de la sociedad colombiana (Salmerón y Gutiérrez, 2012).

De este modo, se evidencia la necesidad de fortalecer las prácticas que permitan la formación de los escolares dadas las dificultades que se detectan en el Proceso de enseñanza aprendizaje en la formación de las habilidades lectoras que afecta a los escolares colombianos del primer grado. Por ello, esta investigación se considera la actividad lúdica como un aporte fundamental para enseñar a leer desde diversas vertientes; en especial, como la condición del ser humano para asumir la vida con goce y disfrute por las acciones que emprende, ayuda en las dimensiones cognitiva, social y emocional, es una actitud muy necesaria, porque produce beneficios.

De modo, que se asume la actividad lúdica como elemento funcional para el desarrollo cognitivo del niño. En la escuela primaria de algunas latitudes de Latinoamérica como Colombia, Perú y Ecuador, entre otros, se denota un patrón de entrega de los resultados en que los escolares aprenden rápidamente el proceso de lectura y de escritura y de forma despersonalizada de los intereses de los escolares, aislado del componente lúdico - creador, se desestima la importancia de la actividad lúdica como componente en doble vía, en el sano esparcimiento y generador en el aprendizaje significativo. No se registra de manera formal y clara la sistematización en la formación de las habilidades lectoras (Gómez, 2015).

En otras investigaciones, se identificó una enseñanza de las vocales y consonantes reflejado en los contenidos, con un método de lectura sintético que resalta los componentes grafo-fónico. Esto ayudó en la mejora de las prácticas académicas, Los escolares manifestaron el deseo de explorar y entender los textos 
porque les era más atractivo; se incorporaron el teatro y la música. No obstante, en esta estrategia no se registran los componentes (etapas, acciones y operaciones) que revelen las formas de organización didáctica, con la utilización de la lúdica como aporte en la formación de habilidades lectoras en este nivel Ardila et al., (2010).

Castro y Marimon (2015) proponen resolver dificultades presentadas frente al aprendizaje de la lectura y la escritura a través de la formación de habilidades y competencias para la lectura en el primer ciclo. Consideran el afianzamiento del código lector basado en el diseño de material didáctico y ambientes de aprendizajes; el desarrollo de los contenidos relacionados con el reconocimiento del alfabeto; su relación con los procesos fonéticos, la producción textual oral, la discriminación visual y auditiva, e interpretación del lenguaje no verbal y la producción escrita.

Piñera (2007), con quien se coincide en este artículo, propone un modelo lúdico para la comprensión lectora en grado sexto de la Educación Básica Primaria. Su modelo está vinculado racionalmente con las actividades curriculares, las reglas y su contenido. Se plantean tareas intelectuales específicas relacionadas con la lectura porque potencian su carácter dinámico en el aprendizaje. Esta investigación se refiere al segundo ciclo de la Educación Básica Primaria.

\section{CONTEXTO, PARTICIPANTES Y METODOLOGÍA}

La metodología se conformó con las técnicas de indagación aplicadas a una muestra de 14 instituciones educativas oficiales del municipio Santiago de Cali en Colombia. Primero, se aplicó la revisión de documentos, específicamente del proyecto educativo institucional y los planes de aula y después se realizó una entrevista a docentes y directivos de las instituciones, tomadas como muestra, se aplicó una encuesta a 31 docentes de primer y segundo grados en dichas instituciones se seleccionaron alrededor de dos docentes por institución. Para indagar sobre el desarrollo de las habilidades lectoras en los escolares se conformó una muestra de 102 escolares de primer grado en la institución Educativa Nuevo Latir. Los resultados se procesaron en SPSS, se usaron estadísticos descriptivos tablas y gráficos y la prueba no paramétrica binomial.

Para valorar, cómo es utilizada la lúdica en el proceso de enseñanza aprendizaje de la lectura en el primer ciclo de la educación básica primaria, en Santiago de Cali, se realizó un diagnóstico del proceso de enseñanza aprendizaje. Para ello se rastreó la existencia de estrategias de aprendizaje centradas en la actividad lúdica como parte del modelo educativo institucional, los métodos y procedimientos que los docentes incluyen en los planes de aula y el lugar que le asignan a la lúdica dentro de dicho proceso. También se indagó sobre el desarrollo de las habilidades lectoras en los escolares.

Para diagnosticar la existencia de estrategias de aprendizaje centradas en la actividad lúdica como parte del modelo educativo institucional, se entrevistaron 14 miembros del consejo académico de la misma cantidad de instituciones educativas oficiales de Educación Básica Primaria en Santiago de Cali. Se indagó, además, el nivel de conocimiento de los integrantes del consejo académico sobre el proceso de enseñanza- aprendizaje de la lectura y su relación con la lúdica para la formación de las habilidades lectoras en los escolares de primer grado.

Para caracterizar el modelo educativo desde el componente método, se seleccionó una muestra de 31 docentes de los grados primero y segundo de la Educación Básica Primaria de instituciones educativas oficiales en Santiago de Cali. El instrumento usado fue la encuesta dirigida a indagar sobre la aplicación de las exigencias de los documentos normativos, los métodos que usan para la enseñanza de la lectura y el empleo de la actividad lúdica. Las respuestas de los docentes se evaluaron en una escala ordinal, en las categorías de básico, medio y alto.

Los 102 escolares de la muestra se extrajeron de una población de 343 que representa el 29,7\% de la población. Están en una edad de los seis a ocho años de edad. 
Las habilidades lectoras se diagnosticaron en cuanto a los componentes: fonético, visual y semántico. El instrumento usado fue una prueba pedagógica. Para facilitar su aplicación se contó con la colaboración de dos practicantes normalistas.

\section{DisCUSIÓN Y RESULTADOS}

En cuanto a la existencia de estrategias de aprendizaje centradas en la actividad lúdica como parte del modelo educativo institucional, el $60 \%$ de los directivos consultados no identifican una estrategia didáctica para la formación de las habilidades lectoras, y valoran su necesidad, privilegiando una posible aplicación de la lectura en situaciones reales de la vida de los escolares, vinculando las experiencias que los maestros puedan tener.

Un 15\% identifica la existencia de estrategias didácticas para la lectura, y refieren que son temporales, su implementación va decayendo y solo al inicio del curso escolar se realizan actividades aisladas.

El 88\% asume que las principales problemáticas que afectan negativamente el proceso de la lectura en el escolar primario es la poca formalización del juego como recurso didáctico y metodológico en el aula de clases.

El 68\% asume que hacen falta orientaciones más para el diseño de una estrategia didáctica con la utilización de la lúdica, que permee los medios de enseñanza, los recursos que creen ambientes de aprendizaje que apliquen la actividad lúdica y la literatura en correspondencia con las edades de la niñez; defienden modelos de, cómo usar la literatura infantil colombiana como parte de la actividad lúdica.

La mayoría de los docentes del Consejo Académico (78\%) identificaron las habilidades más importantes de la lectura, como comprender e interpretar. Apuntan la importancia del desarrollo de la atención y la motivación y asumen que se requieren actividades que ayuden a estimular la conciencia fonológica y la lectura comprensiva mediante distintas situaciones comunicativas. En el quehacer pedagógico reconocen, que se debe garantizar que este tipo de procesos se faciliten al interior de los diversos ciclos.

Así también se realizó una encuesta a 31 docentes que enseñan en el área de lengua castellana en el primer ciclo de la Educación Básica Primaria en el municipio Santiago de Cali. Para su análisis se tienen en cuenta algunas de las preguntas a partir de la encuesta, por parte de los profesores en el proceso de la enseñanza de la lectura y el lugar que le asignan a la lúdica para la formación de las habilidades lectoras. El cuestionario se diseñó preguntas relacionadas con: métodos usados para la enseñanza de la lectura, enseñanza de la lectura alfabética con ilustraciones, las gramaticales y algunas estrategias relacionadas con juegos de dominancia visual auditiva como los bingos lectores, las loterías y los juegos kinestésicos a partir del ritmo de las palabras, representación de los tiempos y formas de las palabras, la narración de texto literarios y la reconstrucción de la misma buscando las palabras añoradas o palabras clave.

La encuesta con los 31 docentes arrojó que más del $50 \%$ de los profesores refieren que no narran anécdotas relacionadas con los rótulos, documentación de historietas, palabras clave y frases clave. Más de un 10\% no usan jeroglíficos ni palabras relacionadas con los rótulos. En el análisis de la enseñanza de la lectura a partir de los documentos normativos, se encontró que más de 50\% de los profesores no consideran importante el análisis de la enseñanza de la lectura a partir de la resolución que norman los indicadores de desempeño en correspondencia con el grado. Seguidamente, se analizó el grupo de preguntas relacionadas con los ambientes de aprendizaje, notándose que menos del $10 \%$ de los profesores no integran la lúdica como concepto de ambiente de aprendizaje en el proceso de enseñanza-aprendizaje de la lectura.

El 83\% afirma que no emplean la actividad lúdica en el Proceso de Enseñanza Aprendizaje de la lectura. El resto plantea, que solo en algunas situaciones usan juegos relacionados con identificar palabras; que hacen competencias entre equipos para seleccionar quien identifica mejor los sonidos o dice el nombre de los objetos mostrados.

Los resultados de la prueba pedagógica aplicada a los escolares de la muestra arrojó los siguientes resultados: El primer ítem dirigido al aspecto fonológico se concretó en la elección de imágenes y palabras en las cuales debían seleccionar la respuesta adecuada con la palabra que tuviese el mismo inicio; otro punto solicitado fue 
el final de la palabra. Esta prueba individual tuvo una duración de media hora; a los escolares se les dispuso las fichas correspondientes, para el desarrollo de prueba. Se evaluó en una escala insuficiente, bajo básico, alto y sobresaliente.

Los resultados alcanzados se ofrecen en la tabla I.

TABLA 1

Resultado de prueba pedagógica según el aspecto fonológico

\begin{tabular}{|l|l|l|l|l|l|}
\hline \multicolumn{2}{|l|}{ Aspecto fonológico } & Frecuencia & Porcentaje & $\begin{array}{l}\text { Porcentaje } \\
\text { válido }\end{array}$ & $\begin{array}{l}\text { Porcentaje } \\
\text { acumulado }\end{array}$ \\
\hline \multirow{4}{*}{ Válidos } & Insuficiente & 10 & 9,8 & 9,8 & 9,8 \\
\cline { 2 - 7 } & Bajo & 65 & 63,7 & 63,7 & 73,5 \\
\cline { 2 - 7 } & Básico & 20 & 19,6 & 19,6 & 93,1 \\
\cline { 2 - 7 } & Alto & 4 & 3,9 & 3,9 & 97,1 \\
\cline { 2 - 7 } & Sobresaliente & 3 & 2,9 & 2,9 & 100,0 \\
\cline { 2 - 7 } & Total & 102 & 100,0 & 100,0 & \\
\hline
\end{tabular}

Fuente: elaboración propia

Estos resultados evidencian que muy pocos escolares alcanzan los niveles de alto y sobresaliente (6,8\%). En el nivel básico solo se encuentra el 19,6\% que sumados a los anteriores ascienden a 26,4. Estos resultados son preocupantes puesto que esta prueba pedagógica se aplicó al finalizar el primer grado, cuando los escolares ya debían haber vencido el objetivo fonológico. El 73,5 de los escolares de la muestra no lograron vencer el objetivo propuesto, por lo que se ubican en los niveles bajo e insuficiente. De modo general, las principales dificultades se concretaron en correlacionar los fonemas vocálicos con el criterio dado. En el Gráfico 1 se ilustran los resultados.

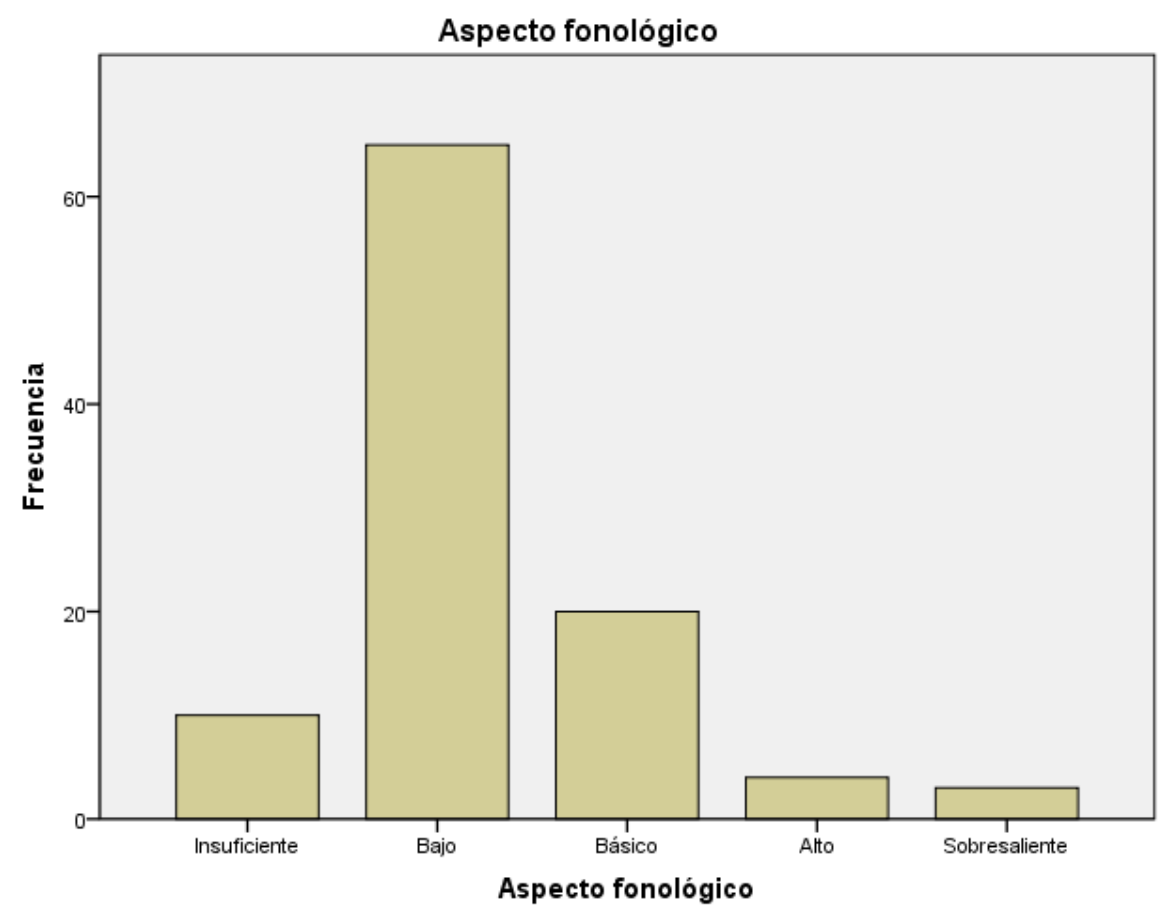

GRÁFICA 1

Resultados en el primer ítem de la prueba pedagógica. Fuente: elaboración propia 
El segundo ítems midió el componente visual y auditivo, el cuestionario se tomó de la subprueba EGRA, allí se le pide al escolar participar en el desafío del abecedario. Cada uno de los escolares debe hacer un ejercicio de identificación de vocales y consonantes que contiene el alfabeto en mayúsculas y minúsculas con un total de 100 signos gráficos.

Se le entregó al niño, el cuadro signos gráficos en cartulina, cada escolar señalaba de izquierda a derecha la cantidad de signos reconocidos. El profesor debe verificar que el escolar reconozca cada uno de los signos de manera oral. La prueba tuvo un tiempo máximo de diez minutos si el escolar así lo requería. La prueba se evalúo en una variable dicotómica (aprobado, desaprobado).

Los estadísticos descriptivos se ofrecen en la Tabla 2

TABLA 2

Estadísticos descriptivos para el segundo ítem. Fuente: Elaboración propia.

\begin{tabular}{|l|l|l|l|l|l|}
\hline \multicolumn{2}{|c|}{ Componente visual auditivo } \\
\hline \multicolumn{2}{|c|}{} & Frecuencia & Porcentaje & Porcentaje válido & Porcentaje acumulado \\
\hline \multirow{3}{*}{ Válidos } & Desaprobado & 92 & 90,2 & 90,2 & 90,2 \\
\cline { 2 - 7 } & Aprobado & 10 & 9,8 & 9,8 & 100,0 \\
\cline { 2 - 6 } & Total & 102 & 100,0 & 100,0 & \\
\hline
\end{tabular}

Considerando una distribución binomial para la variable, se supone según los casos desaprobados lo cual se prueba usando una aproximación normal. Con las hipótesis contra . Considerando un nivel de significación de 0,05. Los resultados procesados en SPSS se ofrecen en la tabla 3.

TABLA 3

Resultados de la prueba no paramétrica binomial

\begin{tabular}{|l|l|l|l|l|l|l|}
\hline \multicolumn{2}{|l|}{ Prueba binomial } & Categoría & N & Proporción observada & Prop. de prueba & Sig. Asintót. (unilateral) \\
\hline \multirow{2}{*}{$\begin{array}{c}\text { Componente } \\
\text { visual } \\
\text { auditivo }\end{array}$} & $\begin{array}{c}\text { Grupo } \\
1\end{array}$ & Aprobado & 10 &, 098 &, 098 &, $548 \mathrm{a}$ \\
\cline { 2 - 7 } & $\begin{array}{c}\text { Grupo } \\
2\end{array}$ & Desaprobado & 92 &, 902 & & \\
\cline { 2 - 7 } & Total & & 102 & 1,000 & & \\
\hline
\end{tabular}

Para el resultado de la prueba se aplicó como estadístico de contraste, la aproximación normal da una probabilidad de que es mucho mayor que 0,05 lo que indica no rechazar es decir que se puede hacer una inferencia a la población de escolares de que existe una probabilidad mayor o igual a 0,902 de desaprobar el componente visual auditivo.

En esta prueba las principales dificultades fueron reconocer el tipo de letras presentadas; hubo dificultades en el reconocimiento de las mayúsculas o minúsculas. Lo que se refleja en la no preparación para la etapa inicial de la lectura (decodificación), en la cual se podría aducir que es por diversos factores externos como el medio cultural, la familia, con limitados ambientes de lectura. Estos resultados indican que los métodos y procedimientos para la formación de habilidades, en sus acciones y operaciones no están conformados teniendo en cuenta los componentes visuales, fonéticos y semánticos.

En el tercer ítem se evaluaron los cuatro componentes. La pregunta se realizó a partir de una visita previa que se realizó con los escolares al zoológico. El escolar debía cambiar algunas palabras por otras que conserven el mismo significado. Esta pregunta se evaluó de manera oral y escrita. Aquí se le entrega al escolar las características de un grupo de animales para señalar el animal que corresponde. Esta prueba también se evaluó 
en una escala dicotómica con el fin de comparar los resultados en la actividad lúdica con los alcanzados en los ítems anteriores. Los resultados se ofrecen en la Tabla 4.

TABLA 4

Resultado del tercer ítem

\begin{tabular}{|l|l|l|l|l|l|}
\hline \multicolumn{6}{|c|}{ Componentes de la lectura con preparación previa } \\
\hline \multicolumn{2}{|c|}{} & Frecuencia & Porcentaje & $\begin{array}{l}\text { Porcentaje } \\
\text { válido }\end{array}$ & $\begin{array}{l}\text { Porcentaje } \\
\text { acumulado }\end{array}$ \\
\hline \multirow{3}{*}{ Válidos } & Desaprobado & 54 & 52,9 & 52,9 & 52,9 \\
\cline { 2 - 6 } & Aprobado & 48 & 47,1 & 47,1 & 100,0 \\
\cline { 2 - 6 } & Total & 102 & 100,0 & 100,0 & \\
\hline
\end{tabular}

Fuente. Elaboración propia.

TABLA 5

Resultados de la prueba no paramétrica binomial para el tercer ítem

\begin{tabular}{|c|c|c|c|c|c|c|}
\hline \multicolumn{7}{|l|}{ Prueba binomial } \\
\hline & & Categoría & N & $\begin{array}{l}\text { Proporción } \\
\text { observada }\end{array}$ & Prop de prueba & $\begin{array}{l}\text { Sig. asintot. } \\
\text { (unilateral) }\end{array}$ \\
\hline \multirow{3}{*}{$\begin{array}{l}\text { Componentes de la lectura } \\
\text { con preparación previa }\end{array}$} & \begin{tabular}{|l|} 
Grupo 1 \\
\end{tabular} & Aprobado & 48 &, 471 & 098 &, $000 \mathrm{a}$ \\
\hline & Grupo 2 & Desaprobado & 54 & .529 & & \\
\hline & Total & & 102 & 1,000 & & \\
\hline
\end{tabular}

Fuente: elaboración propia.

Considerando una distribución binomial para la variable, se supone según los casos desaprobados (se comparó con este valor para poder tener como referencia los resultados del segundo ítem), lo cual se prueba usando una aproximación normal. Con las hipótesis contra se consideró un nivel de significación de 0,05. Para el resultado de la prueba se aplicó como estadístico de contraste la aproximación normal que dio una probabilidad de que es mucho menor que 0,05 lo que indica rechazar Ello implica que existen evidencias significativas de que la probabilidad de desaprobar el ítem con una preparación es menor de 0,902. Es decir que los resultados mejoran cuando se realiza una actividad lúdica previa.

\section{Conclusiones}

La investigación realizada permitió evidenciar que:

En Colombia existe un modelo centrado en competencias para el desarrollo del proceso de enseñanza aprendizaje de la lectura en el primer ciclo de la educación básica primaria.

El modelo colombiano para el desarrollo del proceso de enseñanza aprendizaje de la lectura en el primer ciclo de la educación básica primaria se encuentra contenido en diversos documentos curriculares de nivel nacional como son los lineamientos curriculares, los estándares básicos por competencia y los derechos básicos de aprendizaje. Estos poseen un carácter abierto y flexible dejando a la elección del docente su concreción a nivel de las instituciones educativas y las aulas.

Existen contradicciones entre el modelo colombiano para el desarrollo del proceso de enseñanza aprendizaje de la lectura en el primer ciclo de la educación básica primaria y su concreción en el proyecto educativo institucional y planes de aula de las instituciones educativas oficiales del municipio Santiago de Cali, comunas 13, 14 y 15, donde al hacer el muestreo de los planes de aula y la malla curricular desde la memoria escrita, el $82 \%$ presentan dificultades en el diseño de los objetivos de los ejes temáticos, sus objetivos son expresados en función de los profesores. Tampoco se declara el método de lectura y las estrategias a 
establecer en la praxis pedagógica. En la observación de la clase se denota la prevalencia de la enseñanza de la lectura a través de ejercicios cargados de escritura y transcripción que lleva al escolar al tedio y linealidad en el aprendizaje.

Los docentes del consejo académico identifican dificultades en la elaboración del proyecto educativo institucional en cuanto a la inclusión de estrategias dirigidas al logro de las habilidades lectoras.

Los docentes de aula afirman la existencia de dificultades en cuanto al uso de estrategias y métodos, fundamentalmente de métodos lúdicos para la planificación del proceso de enseñanza aprendizaje de las habilidades lectoras.

La prueba pedagógica aplicada a los escolares de la muestra evidencia dificultades en la decodificación, la significación y la semántica lo que muestra una falta de correspondencia entre las exigencias de los documentos nacionales y los resultados sobre habilidades lectoras alcanzados en la práctica.

\section{ReferenCias BibliográficAS}

Ardila, I., Castaño, A. I., \& Tamayo , G. (2010). Estrategias lúdico pedagógicas para el fortaleciendo de la comprensión lectora.: Fundación universitaria los libertadores- facultad de ciencias de la educación. Arias. Armenia- Quindío. Colombia.

Balmaseda, N. O. (2012). Estado actual de la enseñanza de la lengua, la lectura y la escritura a escala mundial. La Habana: Gente Nueva.

Barboza, P., Delhi, F., \& Peña, G. (2014). El problema de la enseñanza de la lectura en educación primaria, 18(59), 133-142. Venezuela

Cassany, D., Luna, M., \& Sanz, G. (1998). Enseñar lengua (4ta ed). Barcelona: Grao.

Castro, D., \& Marimón, R. (2015). Juguemos con ABC: Estrategia lúdica pedagógica para enseñar a fortalecer los procesos de lectura y escritura en niños de 5 a 7 años. Universidad de Cartagena. Colombia

Castro, J., Robledo,R., Tello, P. (2017). Uso de b-learningen educación primaria para desarrollar la habilidad lectora. Revista de Investigación Educativa de la Escuela de Graduados en Educación. Núm. 14. Recuperado de http:// rieege.tecvirtual.mx/

Cajiao, F., López, I. Betancourt, M., Gómez, L., Tobón, A. Cabrera, A., Gómez, C. (2007). Lineamientos Primer ciclo de educación formal en Bogotá De preescolar a $2^{\circ}$ grado de primaria. Colombia

Delgadillo, C. , \& Chacón, J. (2014). La lúdica como estrategia didáctica para el fortalecimiento de la lectura y la escritura. Bogotá Colombia

Farías, D., \& Rojas, F. (2010). Paradigmas. Retrieved from http://www.scielo.org.ve/scielo

García, G. (2002). Compendio de pedagogía. La Habana: Pueblo y Educación.

Gómez, T. (2015). La actividad lúdica como estrategia pedagógica para fortalecer el aprendizaje en los niños de la Institución educativa Niño Jesús de Praga. Ibagué- Tolima. Colombia

Ministerio de Educación Nacional. (1994). Ley General de educación, Ley 115. Colombia. Congreso de la República de Colombia

Ministerio de Educación Nacional. (2006). Estándares Básicos de Competencias en Lenguaje, Matemáticas, Ciencias y Ciudadanas. Ministerio de Educación Nacional. Colombia. MEN.

Ministerio de Educación Nacional. (2016). Derechos Básicos de Aprendizaje. Ministerio de Educación Nacional. Colombia. MEN.

Ministerio de Educación Nacional. (2015). Malla de aprendizaje de Lenguaje. Ministerio de Educación Nacional. Colombia. MEN.

Piñera, C. (2007). Modelo Lúdico para la comprensión de la Edad de Oro en los escolares de 6to grado. Instituto Superior pedagógico Rafael María de Mendive. Pinar del Rio Cuba.

Salmerón y Gutíerrez (2012). Estrategias de comprensión lectora: Enseñanza y evaluación de Educación primaria. Universidad de Granada. Granada España 
Betty Chalá Molina, et al. Desafíos del modelo colombiano pedagógico para las habilidades lectoras...

Los autores otorgan el permiso a compartir y usar su trabajo manteniendo la autoría del mismo. CC BY-NC 